\title{
RELATIVE AND ABSOLUTE CALIBRATION OF A MULTIHEAD CAMERA SYSTEM WITH OBLIQUE AND NADIR LOOKING CAMERAS FOR A UAS
}

\author{
F. Niemeyer ${ }^{a}{ }^{*}$, R. Schima ${ }^{a}$, G. Grenzdörffer ${ }^{a}$ \\ ${ }^{a}$ Chair for Geodesy and Geoinformatics, Rostock University,18059 Rostock, Germany - [frank.niemeyer] \\ [robert.schima] [goerres.grenzdoerffer] @uni-rostock.de
}

KEY WORDS: multi camera system, oblique camera, calibration, „Australis“, UAS

\begin{abstract}
:
Numerous unmanned aerial systems (UAS) are currently flooding the market. For the most diverse applications UAVs are special designed and used. Micro and mini UAS (maximum take-off weight up to $5 \mathrm{~kg}$ ) are of particular interest, because legal restrictions are still manageable but also the payload capacities are sufficient for many imaging sensors. Currently a camera system with four oblique and one nadir looking cameras is under development at the Chair for Geodesy and Geoinformatics. The so-called "Four Vision" camera system was successfully built and tested in the air. A MD4-1000 UAS from microdrones is used as a carrier system. Light weight industrial cameras are used and controlled by a central computer. For further photogrammetric image processing, each individual camera, as well as all the cameras together have to be calibrated. This paper focuses on the determination of the relative orientation between the cameras with the „Australis“ software and will give an overview of the results and experiences of test flights.
\end{abstract}

\section{INTRODUCTION}

The numbers of different UAV (unmanned aerial vehicle) or UAS (unmanned aerial system) is increasing more and more. UAVs are most often distinguished by weight class, e.g. Blyenburgh \& Co., 2012. In Germany micro and mini UAS with a maximum takeoff weight (MTOW) of up to $5 \mathrm{~kg}$ for micro and $25 \mathrm{~kg}$ for mini UAS respectively are of particular interest, because the legal restrictions are still manageable but also the payload capacities are sufficient for many imaging sensors. The legal framework is defined by the LuftVG and the LuftVO (2012). Because of the possibility to take a camera or a scanning system on board micro and mini UAV become more and more importance in the field of surveying, photogrammetry and remote sensing.

The size of the photographed surface from a UAS depends on altitude, camera or lens and the flight time (battery performance). A multi-camera system with oblique-looking cameras may increase the covered surface area per flight significantly. Furthermore objects will be filmed from different viewing angles and perspectives. There is the possibility to examine the reflection behaviour of object surfaces for certain spectral channels, because object surfaces reflect sunlight from different viewing angles with different intensity. With these results it is possible to calculate the anisotropic behaviour for deriving of the empirical bidirectional reflectance distribution function (BRDF). Interesting information about the vital status of plants can be drawn on the basis of those measurements (Grenzdörffer \& Niemeyer, 2011). Oblique-looking aerial images are also very good with appropriate redundancy for the calculation of a digital surface model (DSM), because there is a more stable geometry between such images. For some aerial surveys oblique looking recordings are essential, e.g. to photograph facades for 3D city models (Linkiewicz, 2013).
Oblique-looking cameras also have disadvantages, for example different scales, different ground sampling distances (GSD) and different surface coverage's within one image. Oblique images from one direction are unsuitable for the generation of an ortho photo because the objects are tilted in the ortho photo and consequently black, invisible areas would occur. Therefore the best solution is a combination of oblique-looking and nadir cameras which allows computing ortho photos, DSM and 3D point clouds with high accuracy.

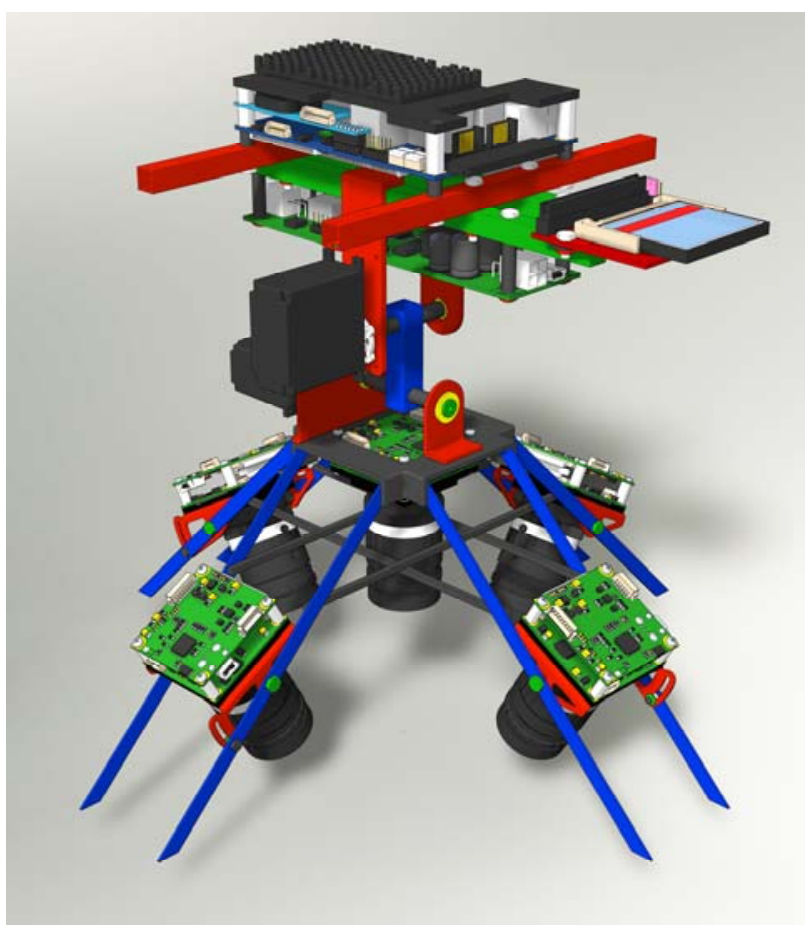

* Corresponding author 
Figure 1: Four Vision System (concept)

Currently a camera system with four oblique and one nadir looking cameras is under development at the Chair for Geodesy and Geoinformatics (Figure 1). The so-called "Four Vision" camera system was successfully built and tested in the air.

\section{METHODS}

\section{1 „Australis“}

It is possible to determine the interior orientation of one or more cameras simultaneously (Photometrix, 2009) with „Australis“ software. For this homologous points of interest in form of coded targets will be automatically detect and identified in every image. The interior orientation of a camera will be calculated with the help of the image coordinates of these targets and a bundle blog adjustment. One target has an area of $4.5 \times 4.5 \mathrm{~cm}^{2}$ and consists of a special constellation of retroreflective circles in front of a black background. It is not necessary to know the correct position in a reference frame. Only the geometry between the targets hasn't to be changed during the image capture process. If there is no information about the coordinates of the targets, the origin of a free XYZ coordinate system will be placed at the perspective centre of the first of the relatively oriented images, and its XZ plane being parallel with the focal plane of the camera at that station. "Australis" has been originally designed for the close range photogrammetry. Therefore the Y-axis looks collinear to the viewing direction. The following images will be oriented in this reference frame, or relative to the first camera. For this a minimum of five targets have to be identified in two or more images. For camera calibration it will be better to have more than six targets in every image of a set of high-and landscapeformat images.

\subsection{Target construction}

A special aluminium construction was created for „Australis“ conditions at the Chair for Geodesy and Geoinformatics. This construction was paste up with over 60 targets and fixed at the ceiling of a lecture room. This construction is geometrically stabile.

\section{3 "Four Vision” Camera System}

In recent years several multi head camera systems with oblique looking cameras were developed for manned aircrafts, e.g. AOS (Aerial Oblique System) from BSF Swissphoto (Wiedemann, 2009) or Quattro DigiCam from Ingenieur-Gesellschaft for Interfaces (Kremer, 2010).

The goal of our research is to develop and to explore a concept of a multi camera system with oblique looking cameras that could be integrated at a micro UAV. The total weight should be somewhat near to $1 \mathrm{~kg}$. A MD4-1000 UAS from microdrones can be used as a carrier system. It has a payload of $1.2 \mathrm{~kg}$. The camera system consists of a top and a substructure. The top part consists of a DC-DC power converter and a minicomputer which controls the cameras and save the images. The subpart consists of one nadir and four oblique looking cameras which can be tilted between $30^{\circ}$ and $60^{\circ}$. This part is moveable in pitch and roll direction via a gimble. The corrections are done by two servomotors. The four cameras are arranged at an angle of $90^{\circ}$ to each other. Therefore the photographed Earth's surface of one image set has the shape of a Maltese cross
(Figure 2). The fifth camera is installed for normal vertical photographs (nadir), see Figure 1.

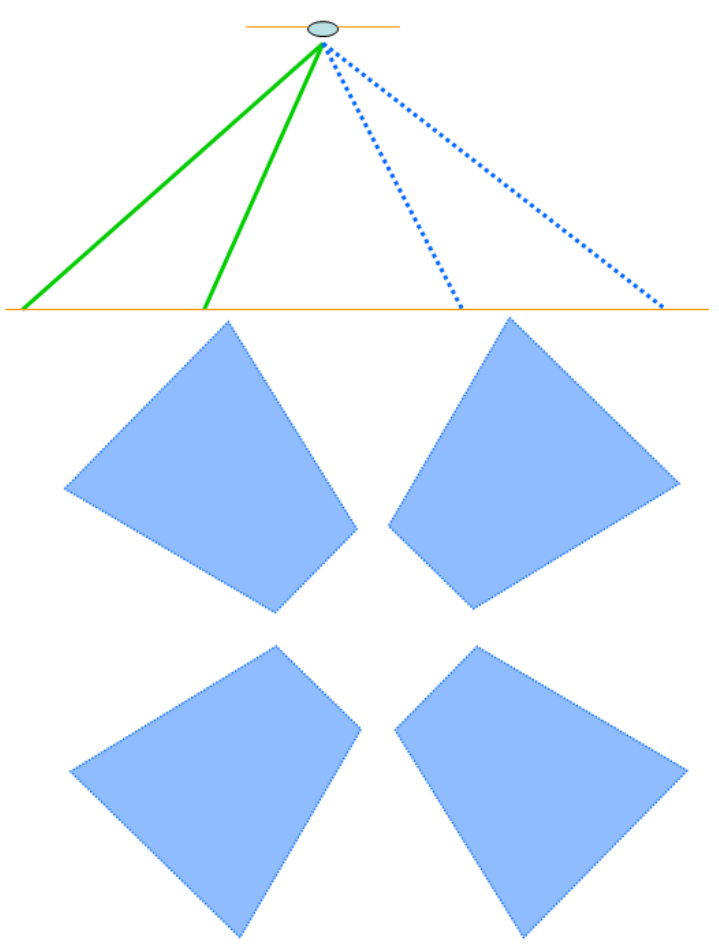

Figure 2: Maltese cross configuration

The cameras of the four vision system are industrial cameras (MV-CS27U) from Crevis and have a $1 / 2$ " Sony CCD sensor. The size of one photo cell is $4.65 \times 4.65 \mu \mathrm{m}^{2}$. The dimension of the sensor is $5.95 \times 4.76 \mathrm{~mm}^{2}$ at a resolution of $1280 \times 1024$ pixels. The camera can trigger up to 15 frames per second and is controlled and powered via a USB 2.0 interface. With the dimensions of $29 \times 29 \times 29 \mathrm{~mm}^{3}$ and a weight of $38 \mathrm{~g}$, the camera is within the weight limits. In addition to the USB interface the MV-CS27U offers two other interfaces for a hardware trigger and a strobe signal or TTL signal for flashlight. With the help of the strobe it is possible to get the exact time of image exposure, because there is a constant delay between exposure time and strobe signal. Finally it is possible to register an image accurately to a certain time and (GPS) position.

Lenses from Fujinon with a focal length of 9 mm (HF9HA-1B) are used for the oblique cameras and a $6 \mathrm{~mm}$ focal lenght (DF6HA-1B) for the nadir camera. As a result, the mapped areas of vertical and oblique images are nearly equal.

\subsection{Controller}

The camera system should make all pictures synchronously. This ensures that the external orientation of one camera can be used to calculate the external orientations of the other four cameras. Software driven synchronous exposures of all cameras cannot be reached with the minicomputer via USB. Because of that a micro controller was built by the Institute of Electronic Appliances and Circuits Department of Computer Science and Electrical Engineering at Rostock University which gives a hardware signal synchronously to all cameras, transforms the analogue strobe signal from cameras to a digital signal and sends the real exposure signal as payload information to the timeline of the MD4-1000. At this time line is based on the GPS 
time. Consequently the images can be co registered and georeferenced to the position of the UAV. During the camera calibration, the synchronous triggering has the side effect that, in contrast to sequential triggering, any external influences (such as vibrations) can't act between the recordings on the system. The controller is programmable. After one trigger signal all images will be saved via USB on minicomputer.

\section{DETERMINATION OF THE RELATIVE} ORIENTATIONS OF THE CAMERAS TO EACH OTHER

The individual cameras have been calibrated and tested (Grenzdörffer et al., 2012) in terms of their comparability. As a result, the principal point positions of the cameras vary quite significantly $( \pm 388 \mu \mathrm{m})$. However, the distortion of the $9 \mathrm{~mm}$ lenses are comparable. Furthermore the vignetting effect was researched for all f-stops of the lenses. F-stop number 4 has the minimum vignetting effect for both $9 \mathrm{~mm}$ and $6 \mathrm{~mm}$ lenses but the two lens types exhibit very different behavior.

To determine the relative orientations of the five cameras to each other, a feature of „Australis“" was being exploited. If „Australis“ has a set of images that depict the same targets with the same geometry, the origin of the reference coordinate system is aligned to focal point of the first camera position and the $\mathrm{Y}$-axis looks in viewing direction.

The experimental setup was arranged so that all cameras of the camera system reflect enough coded targets simultaneously. The camera system was mounted on a tripod to prevent movement during the exposure time. Because the exposure conditions at the test stand did not allow automatic detection of the target in the images, LED lines were attached to each camera as the illumination source and oriented on the camera viewing direction. The coded targets were detected because of their reflection characteristics. After a set of 5 images (one image per camera) was captured, the orientation and position of the camera system were changed. 18 of 20 different positions could be used for further calculations. That means that an adequate number of 18 frames per camera with 56 identified coded targets exist for camera calibration. In a first step all cameras were calibrated and the internal orientations of every camera was stored in a database and fixed for further calculations. For the further calculations the reference system of „Australis" was aligned with the nadir camera. The position of the nadir camera is therefore $(0,0,0)$ and the viewing directions azimuth $=0^{\circ}$, elevation $=0^{\circ}$ and roll angle $=0^{\circ}$.

The images of the nadir camera had therefore to be loaded as the first in the „Australis“ project. After the calculation and saving of the exterior orientations of all five cameras, the order of the first nadir image was changed and the next set was calculated and saved. This resulted in 18 similar orientation parameters for each camera.

Figures 3-6 show the respective position $(X c, Y c, Z c)$ and Figures 7-10 the orientations (azimuth $(\alpha)$, elevation $(v)$, roll angle $(\phi))$ of the oblique-looking cameras of these 18 observation sets in „Australis“" coordinate system.

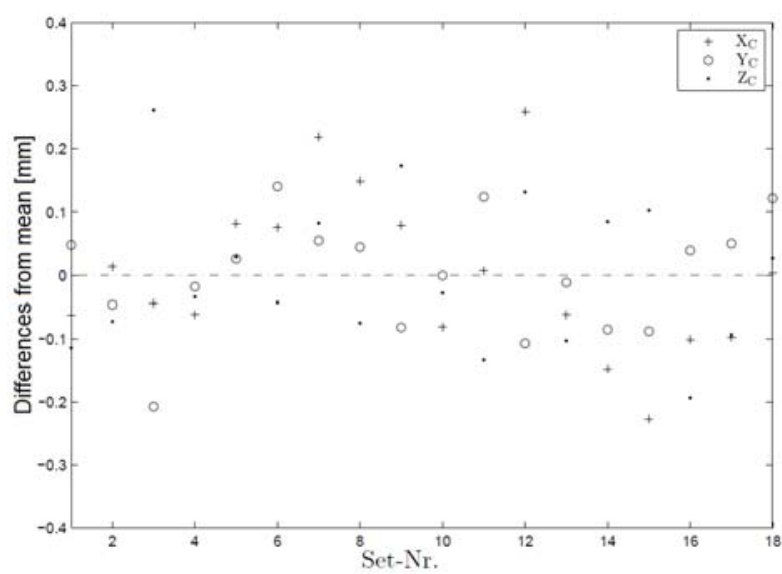

Figure 3: Position accuracy MV-CS27U 1

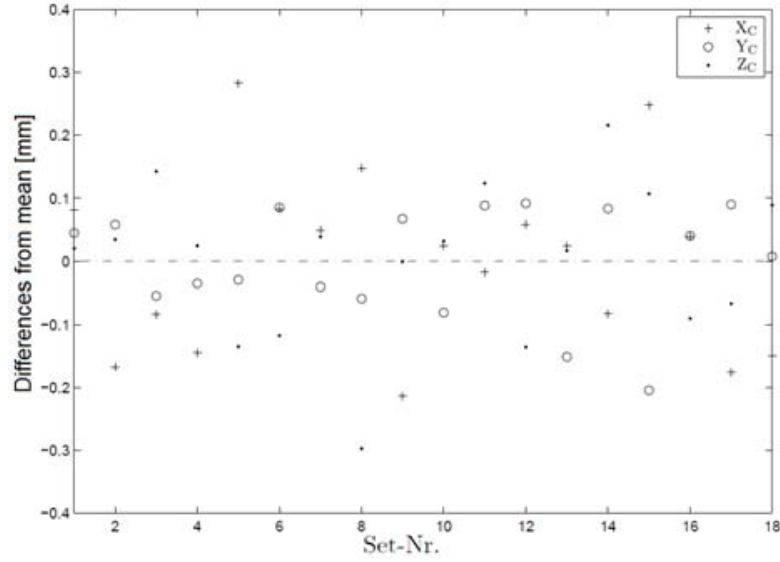

Figure 4: Position accuracy MV-CS27U 2

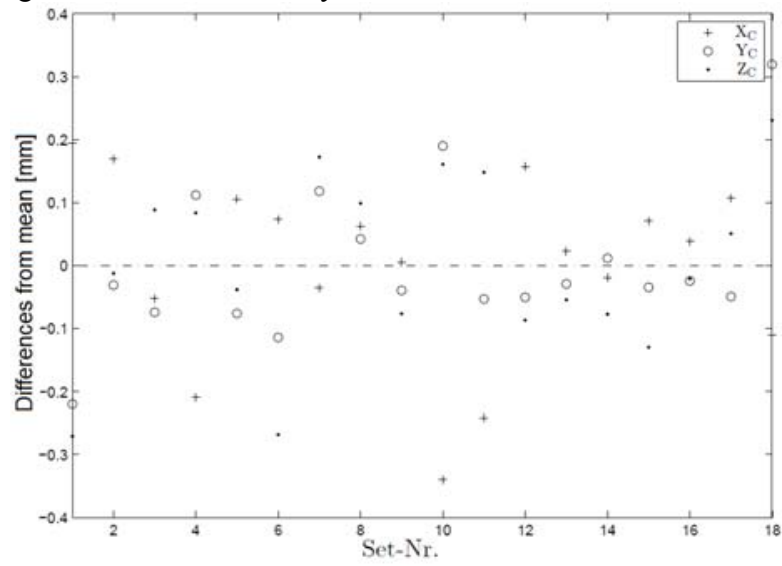

Figure 5: Position accuracy MV-CS27U 3

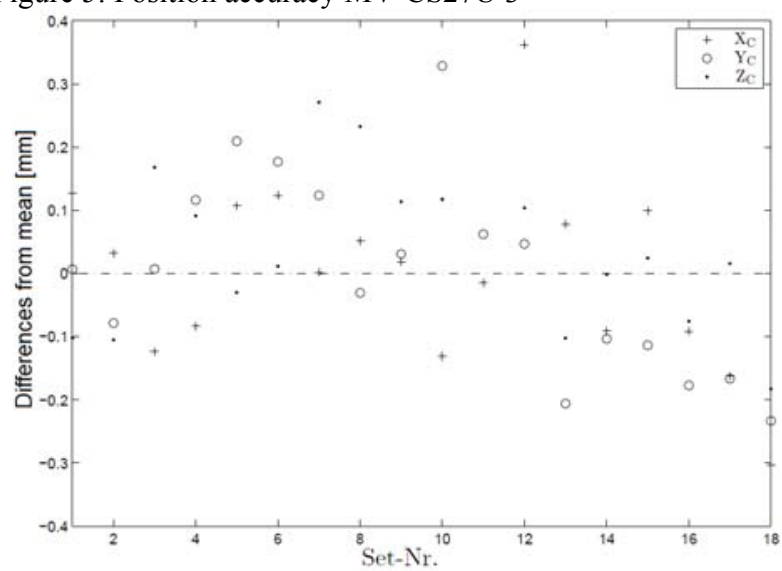

Figure 6: Position accuracy MV-CS27U 4 


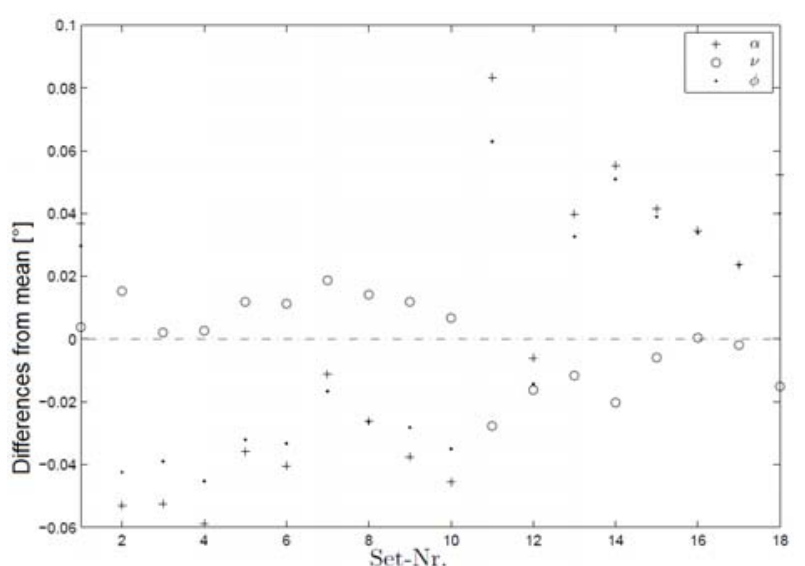

Figure 7: Orientation accuracy MV-CS27U 1

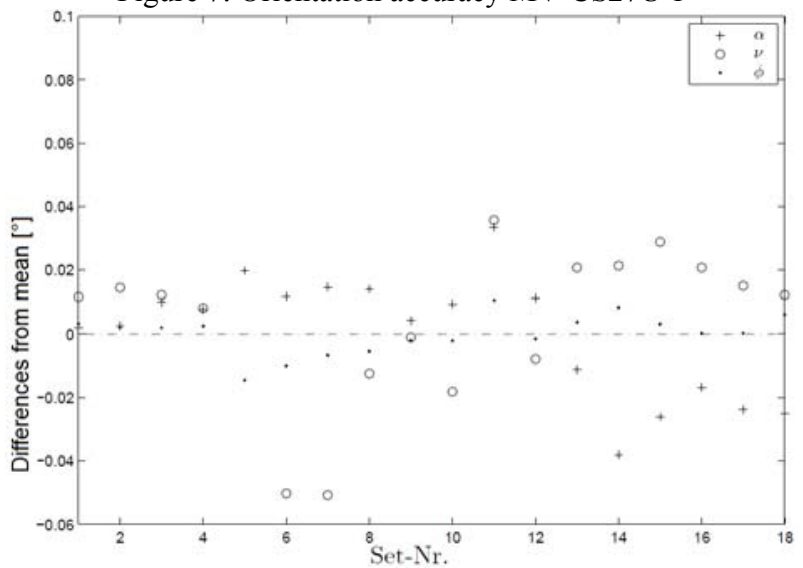

Figure 8: Orientation accuracy MV-CS27U 2

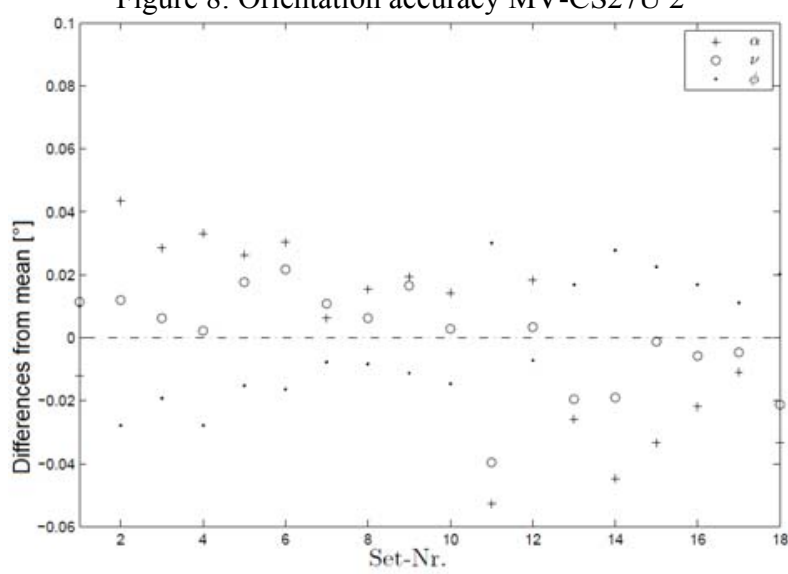

Figure 9: Orientation accuracy MV-CS27U 3

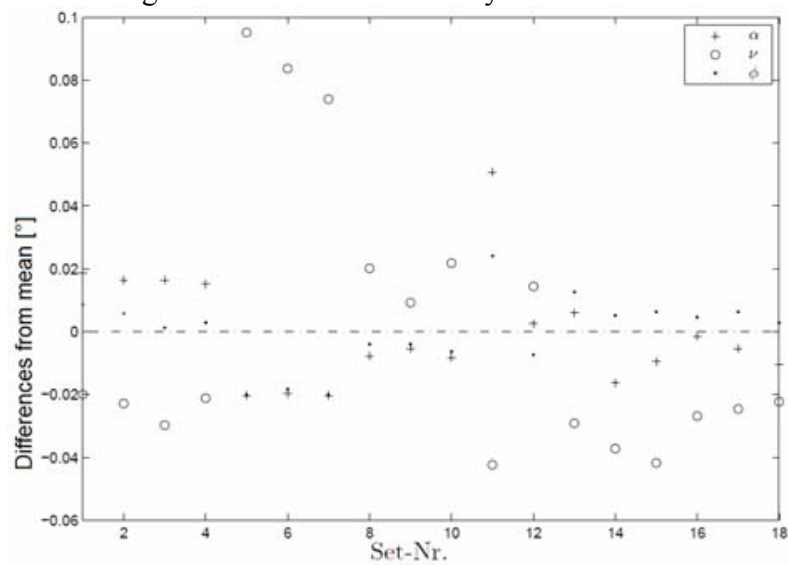

Figure 10: Orientation accuracy MV-CS27U 4
Table 1 gives an overview of the resulting positions and orientations and their mean error $(\sigma)$.

\begin{tabular}{|c|c|c|c|c|}
\hline & $\begin{array}{c}\mathrm{MV}-\mathrm{CS} 27 \mathrm{U} \\
1 \\
\end{array}$ & $\begin{array}{c}\mathrm{MV}-\mathrm{CS} 27 \mathrm{U} \\
2 \\
\end{array}$ & $\begin{array}{c}\mathrm{MV}-\mathrm{CS} 27 \mathrm{U} \\
3 \\
\end{array}$ & $\begin{array}{c}M V-C S 27 U \\
4 \\
\end{array}$ \\
\hline$x[\mathrm{~mm}]$ & -1.38 & -76.49 & -0.25 & 73.25 \\
\hline$\sigma_{x}[\mathrm{~mm}]$ & 0.13 & 0.14 & 0.15 & 0.15 \\
\hline $\mathrm{y}[\mathrm{mm}]$ & 34.10 & 31.44 & 31.74 & 34.62 \\
\hline$\sigma_{y}[\mathrm{~mm}]$ & 0.09 & 0.09 & 0.12 & 0.15 \\
\hline $\mathrm{z}[\mathrm{mm}]$ & -75.22 & -0.01 & 75.07 & -0.25 \\
\hline$\sigma_{z}[\mathrm{~mm}]$ & 0.12 & 0.12 & 0.14 & 0.18 \\
\hline Azimut $\left[{ }^{\circ}\right]$ & 2.20 & -29.50 & -0.62 & 32.05 \\
\hline$\sigma_{\text {Azimut }}\left[{ }^{\circ}\right]$ & 0.05 & 0.02 & 0.03 & 0.02 \\
\hline Elev. $\left[{ }^{\circ}\right]$ & 29.68 & 0.07 & -31.74 & 0.20 \\
\hline$\sigma_{\text {Elev. }}\left[{ }^{\circ}\right]$ & 0.01 & 0.03 & 0.02 & 0.04 \\
\hline Roll $\left[{ }^{\circ}\right]$ & 2.11 & -89.51 & -177.62 & 90.62 \\
\hline$\sigma_{\text {Roll }}\left[{ }^{\circ}\right]$ & 0.04 & 0.01 & 0.02 & 0.01 \\
\hline
\end{tabular}

Table 1: Exterior Orientation of oblique looking cameras

Because the coordinate system in "Australis" is defined that the viewing direction of the camera is oriented in the $Y$ direction, the camera system appears tilted by $90^{\circ}$ (Figure 11). But the camera system looks downwards during the flight (Figure 12).
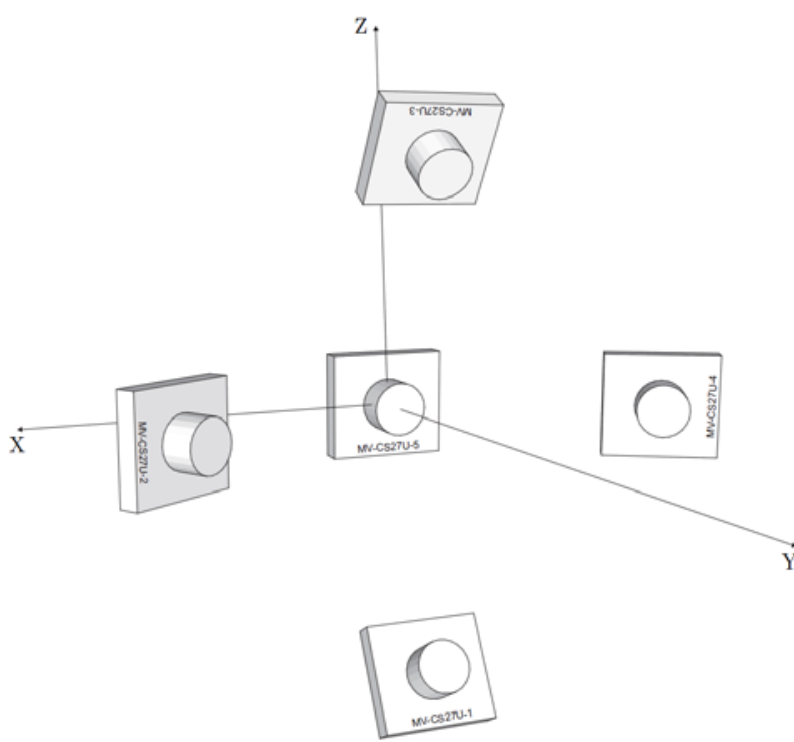

Figure 11: „Australis“ coordinate system 


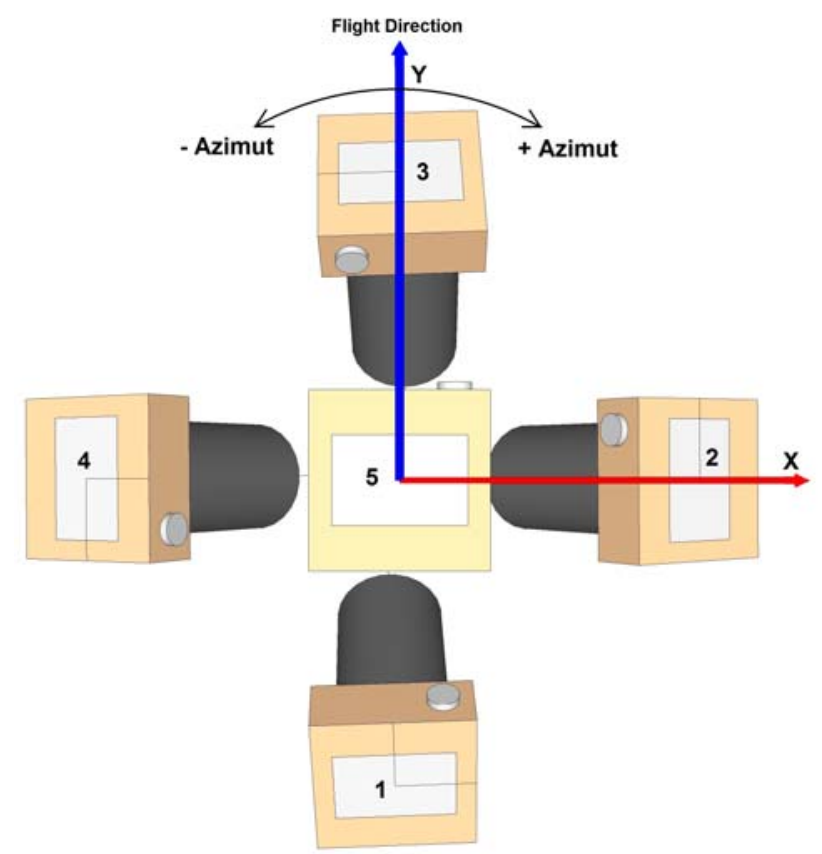

Figure 12: Normal flight coordinate system

The converted camera orientations for the normal coordinate system during the flight can be found in Table 2.

\begin{tabular}{|c|c|c|c|c|}
\hline & $\begin{array}{c}\text { MV-CS27U } \\
1 \\
\end{array}$ & $\begin{array}{c}\mathrm{MV}-\mathrm{CS} 27 \mathrm{U} \\
2 \\
\end{array}$ & $\begin{array}{c}\mathrm{MV}-\mathrm{CS} 27 \mathrm{U} \\
3 \\
\end{array}$ & $\begin{array}{c}\text { MV-CS27U } \\
4 \\
\end{array}$ \\
\hline Azi.' [ $\left.{ }^{\circ}\right]$ & -2.11 & -89.93 & 177.622 & 89.80 \\
\hline Elev.' $\left[{ }^{\circ}\right]$ & -60.32 & -60.50 & -58.26 & -57.95 \\
\hline $\operatorname{Roll}^{\prime}\left[{ }^{\circ}\right]$ & 2.20 & 0.49 & -0.62 & 0.62 \\
\hline
\end{tabular}

Table 2: Viewing directions of oblique looking cameras in relation to nadir camera for a normal flight coordinate system

\section{RESULTS AND DISCUSSION}

The results of the relative orientations of the cameras to each other were determined with an accuracy of camera positions between $0.09 \mathrm{~mm}$ to $0.18 \mathrm{~mm}$ and the camera orientations very accurate from $0.01^{\circ}$ to $0.05^{\circ}$. Based on figures 3 to 10 it could be clearly shown how low the variability of the measurement series is. The relative orientation of the cameras to each other under static conditions can be regarded as constant despite of repeated changed position of the whole system.

An experimental setup was developed, which allows the calibration of multi-camera systems. The proposed method provides not only robust but also sufficiently accurate results especially for photogrammetric issues. The method is requires also a comparatively low experimental and temporal effort. Future tests under dynamic flight conditions will show whether the relative orientations are sufficiently stable to simplify further photogrammetric data processing. Because if the relative position of the cameras is known, it is sufficient to orient only the nadir looking camera classic with direct or indirect methods. The oblique-looking cameras are then subsequently oriented relative with the help of the known lever arms and angle relation, Jacobsen, 2009.

\section{ACKNOWLEDGEMENT}

The presented research "PFIFFikus" is supported by TBI Technologie-Beratungs-Institut $\mathrm{GmbH}$ of the Ministry of Economics, Labour and Tourism Mecklenburg-Vorpommern under the grant number V230-630-08-TFMV-F041.

\section{REFERENCES}

Blyenburgh \& Co. [ed], 2012. 2012 RPAS Yearbook - RPAS: The Global Perspective, Blyenburgh \& Co., Den Haag.

Eisenbeiss, H., 2009. UAV photogrammetry, Dissertation, Eidgenössische Techn. Hochsch, Zürich, Zürich.

Federal Ministery of Transport, 10.08.1963.: LuftverkehrsOrdnung - LuftVO, (=http://www.gesetze-im-internet.de/ luftvo/BJNR006520963.html) (10.10.2012).

Grenzdörffer, G., Niemeyer, F., 2011. UAV Based BRDFMeasurements of Agricultural Surfaces with PFIFFIKUS. In: Eisenbeiss, H. et al. [eds], Int. Arch. Photogramm. Remote Sens. Spatial Inf. Sci. Proceedings of the International Conference on Unmanned Aerial Vehicle in Geomatics (UAVg), Zürich.

Grenzdörffer, G., Niemeyer, F., Schmidt, F., 2012. Development of Four Vision Camera System for a Micro UAV. ISPRS (Int. Arch. Photogramm. Remote Sens. Spatial Inf. Sci.), ISPRS Congress 2012, Melbourne, Australia, XXII ISPRS, S. 369-374.

Jacobsen, K., 2009. Geometry of vertical and oblique image combinations. In: Maktav, D. [ed], Remote Sensing for a Changing Europe. Proceedings of the 28th Symposium of the European Association of Remote Sensing Laboratories, Istanbul, Turkey, 2-5 June 2008, IOS Press, Amsterdam.

Kremer, J., 2010. The Quattro DigiCAM - IGI's Versatile Aerial Camera System for Various Aerial Imaging Tasks. In: Seyfert, E. [ed], DGPF-Publikationen, Deutsche Gesellschaft für Photogrammetrie, Fernerkundung und Geoinformation (DGPF) e.V., Oldenburg, S. 623-630.

Linkiewicz, M., 2013. Extraktion von senkrechten Fassadenebenen aus 3D Punktwolken von Schrägluftbildern. In: Seyfert, E. [ed], DGPF-Publikationen, Deutsche Gesellschaft für Photogrammetrie, Fernerkundung und Geoinformation (DGPF) e.V., Oldenburg,S. 354-362.

Photometrix, 2009. „Australis“, Download link http://www.photometrix.com.au/support.html. (11.03.2012).

Wiedemann, A., 2009. Photogrammetrische Schrägluftbilder mit dem Aerial Oblique System AOS. In: Seyfert, E. [Hrsg.], DGPF-Publikationen, Deutsche Gesellschaft für Photogrammetrie, Fernerkundung und Geoinformation (DGPF) e.V., Oldenburg, S. 1-8. 